\title{
Dust properties and distribution in dwarf galaxies
}

\author{
Ute Lisenfeld $^{1,2}$, Monica Relaño ${ }^{1,2}$, José Vílchez ${ }^{3}$, Eduardo Battaner ${ }^{1}$ \\ and Israel Hermelo ${ }^{1}$ \\ ${ }^{1}$ Universidad Granada, Spain \\ email: ute@ugr.es, battaner@ugr.es \\ ${ }^{2}$ Institute of Astronomy, University of Cambridge, UK \\ email: mrelano@ast.cam.ac.uk \\ ${ }^{3}$ Instituto de Astrofísica de Andalucía, Granada, Spain \\ email: jvm@iaa.es
}

\begin{abstract}
We present a study of the extinction, traced by the Balmer decrement, in HII regions in the dwarf galaxies NGC 1569 and NGC 4214. We find that the large-scale extinction around the most prominent HII regions in both galaxies forms a shell in which locally the intrinsic extinction can adopt relatively high values $\left(A_{V}=0.8-0.9\right.$ mag $)$ despite the low metallicity and thus the low overall dust content. The small-scale extinction (spatial resolution $\sim 0.3$ ") shows fluctuations that are most likely due to variations in the dust distribution. We compare the distribution of the extinction to that of the dust emission, traced by Spitzer emission at 8 and $24 \mu \mathrm{m}$, and to the emission of cold dust at $850 \mu \mathrm{m}$. We find in general a good agreement between all tracers, except for the $850 \mu \mathrm{m}$ emission in NGC 4214 which is more extended than the extinction and the other emissions. Whereas in NGC 1569 the dust emission at all wavelengths is very similar, NGC 4214 shows spatial variations in the 24 -to- $850 \mu \mathrm{m}$ ratio.

We furthermore compared the $24 \mu \mathrm{m}$ and the extinction-corrected $\mathrm{H} \alpha$ emission from HII regions in a sample of galaxies with a wide range of metallicities and found a good correlation, independent of metallicity. We suggest that this lack of dependence on metallicity might be due to the formation of dust shells with a relatively constant opacity, like the ones observed here, around ionizing stars.
\end{abstract}

Keywords. ISM: dust, ISM: extinction, galaxies: ISM, galaxies: individual (NGC 1569), galaxies: individual (NGC 4214), galaxies: dwarf

\section{Introduction}

Interstellar dust can be studied via its emission and also via the extinction that it causes. Each method has its advantages and difficulties. The most common way of obtaining the extinction of the light coming from HII regions is based on the comparison of the $\mathrm{H} \alpha$ and $\mathrm{H} \beta$ recombination line fluxes. A major advantage of this method is the high spatial resolution achieved, determined by the resolution of the optical images. It is, however, difficult to derive the distribution of the dust from extinction maps because the relative distribution of the dust and the gas plays a major role and because maps of the $\mathrm{H} \alpha / \mathrm{H} \beta$ ratio are biased towards low-extinction regions.

Alternatively, the dust can be studied via its emission, which depends on the amount of dust, the type of grains and the dust temperature determined by the interstellar radiation field (ISRF). Although very different models for the interstellar dust exist (see, e.g., Zubko et al. 2004 and references therein), they generally need to include three types of grains: big grains that are in thermal equilibrium with the ISRF, very small grains (VSGs) that are stochastically heated and are necessary to explain the mid-infrared emission 
and Polycyclic Aromatic Hydrocarbons (PAHs) to explain the line features in the $8 \mu \mathrm{m}$ range. So far there have been two major problems for studies of the dust emission: The lack of images at a high spatial resolution and the lack of submillimeter data probing the cold dust. Fortunately, the situation is improving. The Spitzer satellite has for the first time provided images of high spatial resolution in the mid-infrared regime where the emission is dominated by PAHs and VSGs. In the near future, the satellites Herschel and Planck will provide a large database for the submillimeter emission of galaxies.

We present a study of the dust extinction (based on the Balmer decrement) and emission in two nearby, starbursting, dwarf galaxies, NGC 1569 and NGC 4214. The goals are to derive the small and large-scale structure of the dust extinction and to compare it to the dust emission at various wavelengths, tracing different types of dust grains and temperatures. This study will allow us to derive information about the dust distribution and variations of the dust temperature and of grain types.

NGC 1569 and NGC 4214 have been chosen in order to study the role of the metallicity and active star formation for the dust. Both galaxies have a low metallicity of $12+\log (\mathrm{O} / \mathrm{H})=8.2$ (Kobulnicky \& Skillman 1997, Armus et al. 1989) and a similar distance (2.2 Mpc to NGC 1569, Israel 1988, and 2.9 Mpc to NGC 4214, Maíz-Apellániz et al. 2002). They both harbour large HII complexes, and host one (NGC 4214) or two (NGC 1569) Super Star Clusters (SSCs), in the middle of large expanding H $\alpha$ shells (Martin 1998).

\section{Extinction maps}

The extinction maps $\left(\mathrm{A}_{H \alpha}\right)$ were calculated using $\mathrm{H} \alpha$ and $\mathrm{H} \beta$ images obtained with the WFPC2 on the Hubble Space Telescope and following the method explained in Caplan \& Deharveng (1986). In NGC 1569, an extinction map of the galaxy was obtained in two different regimes characterized by different physical conditions: extinction within the HII regions and extinction of the diffuse ionized gas (see Relaño et al. 2006, for a detailed explanation of the procedure). In NGC 4214, the extinction was only calculated for the HII regions and the diffuse ionized gas was excluded. In both galaxies, we furthermore excluded regions with a low $\mathrm{H} \beta$ equivalent width (values below $75 \AA$ ) because of the possible stellar absorption of $\mathrm{H} \beta$ due to evolved stars.

\subsection{Large-scale distribution}

In Fig. 1 we show the resulting extinction maps overlaid with the $\mathrm{H} \alpha$ emission distribution, both smoothed to a resolution of 6 " in order to make the large-scale distribution visible. We see various interesting features that are in common in both galaxies:

- The highest values in extinction form a shell structure around the brightest HII regions. The shells are also visible in $\mathrm{H} \alpha$ but much clearer in the extinction. They spatially correlate with expanding supershells catalogued by Martin (1998) (NGC 1569-C, respectively NGC 4214-A).

- The values of the intrinsic extinction (i.e. after subtracting the foreground Galactic extinction) in the shells are $\mathrm{A}_{H \alpha}=0.3-0.7$ mag in both galaxies. These value are relatively high for these low-metallicity galaxies, indicating that locally high-opacity regions can exist, in spite of the globally low dust content.

We suggest that the shell-like extinction structure has been formed by a cumulative deposit of dust at the boundary of the supershell. In NGC 1569, an analysis of the energies involved shows that the stellar winds coming from SSC A are able to produce the expanding shell and to sweep up the dust mass contained in it (Relaño et al. 2006). 


\subsection{Small scale distribution}

In the 0.3 " resolution $\mathrm{H} \alpha / \mathrm{H} \beta$ image a very clumpy structure is visible (see Fig. 3 of Relaño et al. 2007 for NGC 1569). In both galaxies, the fluctuations are clearly above the level expected from the noise in the $\mathrm{H} \alpha$ and $\mathrm{H} \beta$ images. We suggest that these fluctuations in $\mathrm{H} \alpha / \mathrm{H} \beta$ can be related to opacity inhomogeneities in the HII regions, most likely due to a clumpy dust distribution.

\section{Comparison of the dust extinction and emission}

In Fig. 2 we show a comparison of the extinction and dust emission at $8 \mu \mathrm{m}, 24 \mu \mathrm{m}$ and $850 \mu \mathrm{m}$. We find a generally good, but not always perfect, agreement between the $8 \mu \mathrm{m}$ and $24 \mu \mathrm{m}$ emission and the dust extinction. There is a trend for the $24 \mu \mathrm{m}$ emission (in NGC 1569 also for the $8 \mu \mathrm{m}$ emission) to be shifted towards the inner side of the extinction shell. This can be due to a combination of two effects: Towards the inner side of the HII region the dust is hotter and thus emits more strongly at $24 \mu \mathrm{m}$. In addition, a geometric effect can explain the displacement of the emission peaks: if the dust accumulates in the outer region of the ionized shell a line of sight in this direction will trace a region of higher opacity than further inside the HII region, where more dust emission is seen due to the long line of sight.

When comparing the dust extinction to the emission of the cold dust at $850 \mu \mathrm{m} \mathrm{ob-}$ served with SCUBA at the James Clerk Maxwell Telescope (Lisenfeld et al. 2001, Kiuchi et al. 2004), we find a different distribution in both objects: in NGC 1569 the distribution at $850 \mu \mathrm{m}$ is very similar to that of $\mathrm{H} \alpha$, to the dust extinction, as well as to the dust emission at 8 and $24 \mu \mathrm{m}$. Within the spatial resolution of $15 \%$, the peak of the $850 \mu \mathrm{m}$ emission agrees with the distribution of all of these tracers and with the dust extinction. In NGC 4214, on the other hand, only part of the $850 \mu \mathrm{m}$ emission coincides with the dust shell, and it is also different from the dust emission at $8 \mu \mathrm{m}$ and $24 \mu \mathrm{m}$. In the southern HII region, there are two marginally resolved peaks of the $850 \mu \mathrm{m}$ emission close to peaks in the extinction and the $\mathrm{H} \alpha$ emission. In the north, part of the $850 \mu \mathrm{m}$ emission
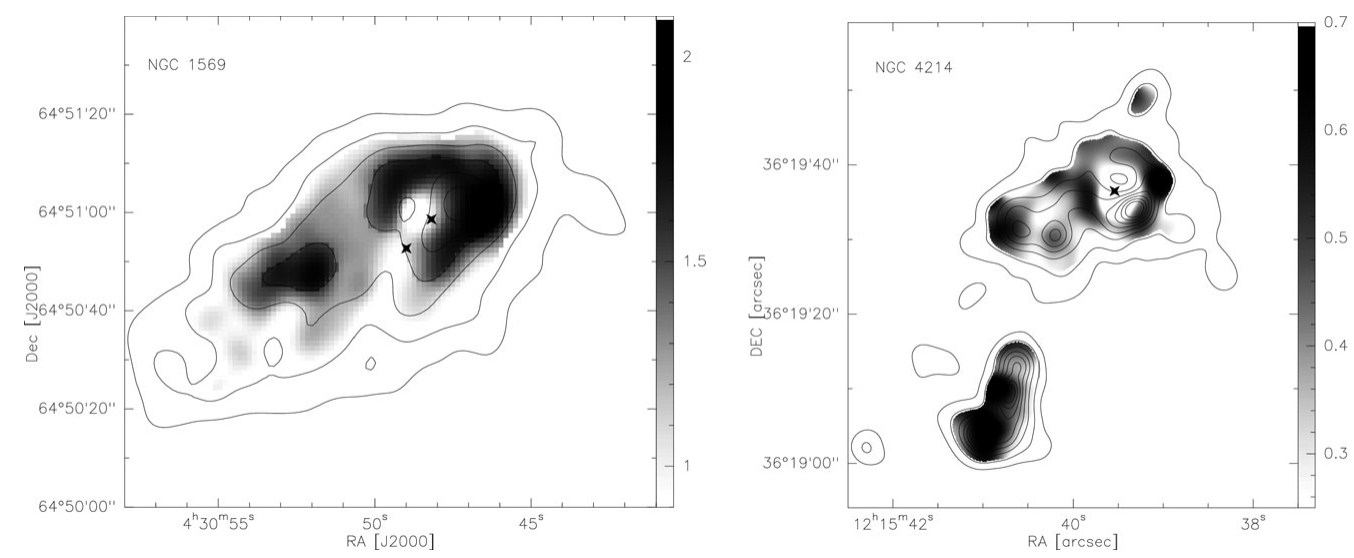

Figure 1. Total (i.e. intrinsic plus Galactic foreground) extinction, $A_{H \alpha}$, (color), overlaid on the emission of $\mathrm{H} \alpha$ (contours) for (left) NGC 1569 and (right) NGC 4214. The resolution of both images is 6". The Galactic foreground extinction is about $A_{H \alpha}=1.3 \mathrm{mag}$ for NGC 1569 (Kobulnicky \& Skillman 1997) and $A_{H \alpha}=0.05$ mag for NGC 4214 (Schlegel et al. 1998). The crosses indicate the positions of the SSCs A and B in NGC 1569, and SSC I-As in NGC 4214. 

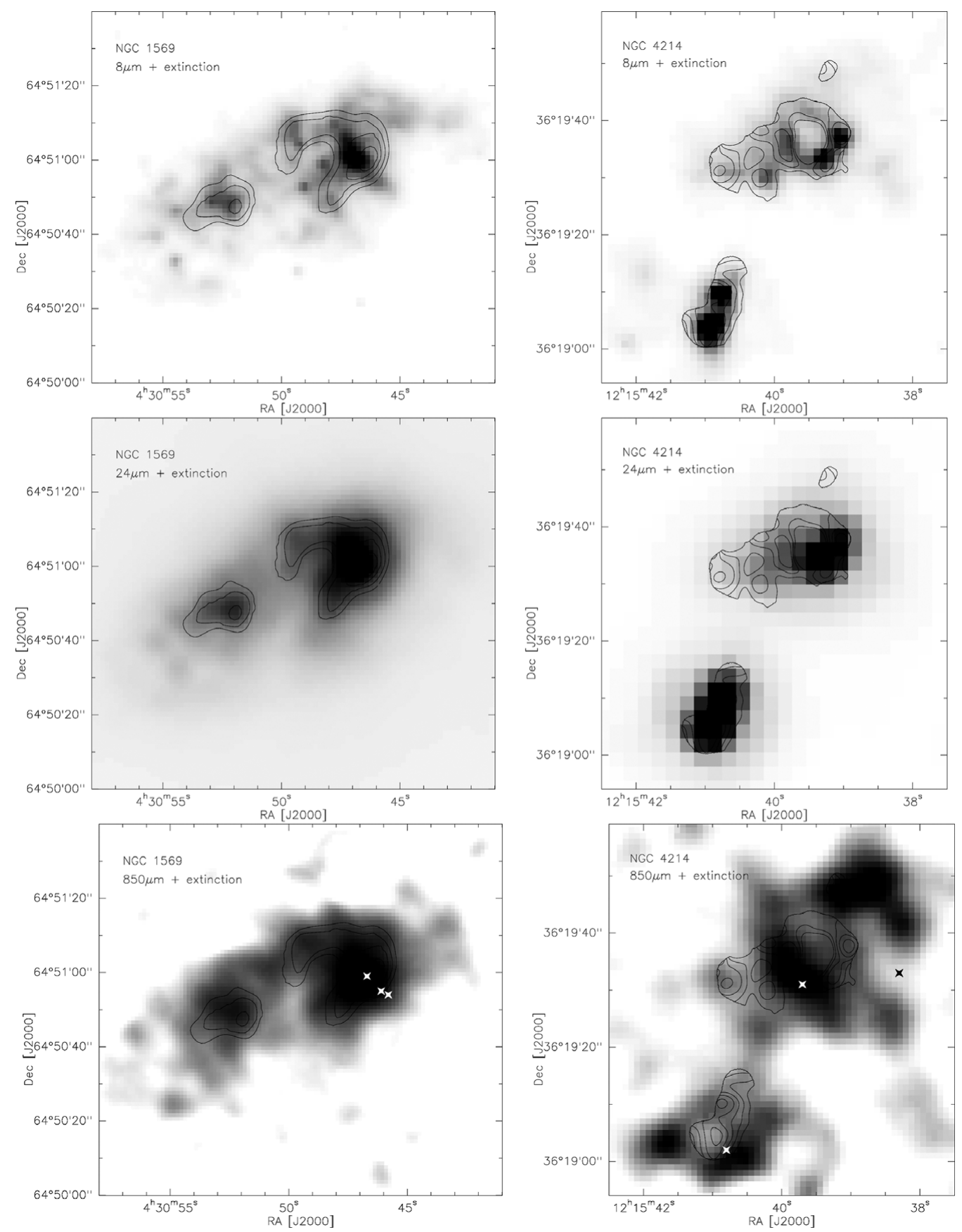

Figure 2. Intrinsic extinction (contours levels at 0.1, 0.3, 0.5 and 0.7 mag for NGC 1569 and at $0.25,0.45$ and $0.65 \mathrm{mag}$ for NGC 4214), overlaid on the emission of 8,24 and $850 \mu \mathrm{m}$ (colour) for NGC 1569 (left) NGC 4214 (right). The 8 and $24 \mu \mathrm{m}$ maps are from the Spitzer archive, and the $850 \mu \mathrm{m}$ maps are observed with SCUBA at the James Clerk Maxwell Telescope. The spatial resolution is 6" (extinction and $24 \mu \mathrm{m}), 1.4 "(8 \mu \mathrm{m})$ and $15 "(850 \mu \mathrm{m})$. The crosses in the lowest panels indicate the positions of the strongest peaks in the $\mathrm{CO}(1-0)$ distribution (Taylor et al. 1999 for NGC 1569 and Walter et al. 2001 for NGC 4214). 
spatially coincides with the extinction shell and the $24 \mu \mathrm{m}$ emission, but the $850 \mu \mathrm{m}$ emission extends further northwest than any other emission considered here. Thus, in contrary to NGC 1569 , NGC 4214 shows very strong variations in the 24 -to- $850 \mu \mathrm{m}$ ratio, indicating the presence of local variations of the dust temperature or the grain composition, and in particular the presence of cold dust in the northwestern part.

In both galaxies there is a close relation between the molecular gas and the emission at $850 \mu \mathrm{m}$ : in NGC 1569 giant molecular clouds are found close to the peak of the $850 \mu \mathrm{m}$ emission. In NGC 4214, on the contrary, the correspondence is not complete (see Fig. 2). In particular, towards the northwest no molecular gas has been found in spite of strong $850 \mu \mathrm{m}$ emission, only atomic gas (Walter et al. 2001) is present.

\section{The $24 \mu \mathrm{m}$ emission as a SF tracer in low-metallicity galaxies}

The good agreement between the $24 \mu \mathrm{m}$ and $\mathrm{H} \alpha$ emission has been found in several other studies in different kind of galaxies (Calzetti et al. 2005, 2007, Pérez-Gonzalez et al. 2006) and it is the base for the use of the $24 \mu \mathrm{m}$ emission as a tracer for star formation (Calzetti et al. 2007, Kennicutt et al. 2007). Relaño et al. (2007) have shown by comparing $24 \mu \mathrm{m}$ and extinction-correced $\mathrm{H} \alpha$ emission integrated over individual HII regions in a sample of galaxies spanning a wide metallicity range (from $12+\log (\mathrm{O} / \mathrm{H})=$ 7.2 to 9.1 ) that the correlation between both emissions does not depend on metallicity. Only when considering the total emissions (i.e. the emission from HII regions plus the diffuse emission), a trend with metallicity was found.

This result is, at first sight, surprising because the $24 \mu \mathrm{m}$ emission depends on the dust content which is directly related to the metallicity. The most likely reason of the observed constancy of the $\mathrm{H} \alpha / 24 \mu \mathrm{m}$ ratio of HII regions is the formation of a dust shell like the ones observed in NGC 1569 and NGC 4214. In these shells, the opacity might be relatively constant and not strongly dependent on the metallicity, due to the existence of a lower threshold for the accumulation of dust in HII regions in order to support large $\operatorname{SFR}\left(\sim 10^{-4}-10^{-1} \mathrm{M}_{\odot} \mathrm{yr}^{-1}\right)$.

\section{References}

Armus, L., Heckman, T. M., \& Miley, G. K., 1989, ApJ, 347, 727

Calzetti, D., Kennicutt, R. C., \& Bianchi., L., et al., 2005, ApJ, 633, 871

Calzetti, D., Kennicutt, R. C., \& Engelbracht, C. W., 2007, ApJ 666, 870

Caplan, J. \& Deharveng, L, 1986, A\&A, 155, 297

Israel, F. P., A\&A, 194, 24

Kiuchi, G., Ohta, K., Sawicki, M., \& Allen, M., 2004, ApJ, 128, 2743

Kobulnicky, H. A. \& Skillman, E., 1997, ApJ, 489, 636

Lisenfeld, U., Israel, F. P., Stil, J. M., \& Sievers, A., 2002 A\&A, 382, 860

Maíz-Apellániz, J., Cieza, L., \& MacKenty, J. W., 2002, AJ, 124, 1601

Martin, C. L., 1998, ApJ, 506, 222

Pérez-González, P. G., Kennicutt, R. C., \& Gordon, K. D., 2006, ApJ, 648, 987

Relaño, M., Lisenfeld, U., Vílchez, J. M., \& Battaner, E., 2006, A\&A, 452, 413

Relaño, M., Lisenfeld, U., Pérez-González, P. G., Vílchez, J. M., \& Battaner, E., 2006, A\&A 667, L141

Schlegel, D. J., Finkbeiner D. P., \& Davis, M., 1998, ApJ, 500, 525

Walter, F., Taylor, C. L., Hüttemeister, S., Scoville, N., \& McIntyre, V., 2001, ApJ, 121, 727

Zubko, V., Dwek, E., \& Arendt, R. G., ApJS, 152, 211

Taylor, C. L., Hüttemeister, S., KLein, U., \& Greve, A., 1999, A\&A, 349, 424 